\title{
Concerted evolution of male and female display traits in the European corn borer, Ostrinia nubilalis Jean-Marc Lassance* and Christer Löfstedt
}

\author{
Address: Department of Ecology, Lund University, S-22362, Lund, Sweden \\ Email: Jean-Marc Lassance* - jean-marc.lassance@ekol.lu.se; Christer Löfstedt - christer.lofstedt@ekol.lu.se \\ * Corresponding author
}

Published: 3 March 2009

BMC Biology 2009, 7:10 doi:10.1186/1741-7007-7-10
Received: 8 September 2008

Accepted: 3 March 2009

This article is available from: http://www.biomedcentral.com/I74/-7007/7//0

(C) 2009 Lassance and Löfstedt; licensee BioMed Central Ltd.

This is an Open Access article distributed under the terms of the Creative Commons Attribution License (http://creativecommons.org/licenses/by/2.0), which permits unrestricted use, distribution, and reproduction in any medium, provided the original work is properly cited.

\begin{abstract}
Background: Sexual reproduction entails the encounter of the sexes and the multiplicity of rituals is parallel to the diversity of mating systems. Evolutionary mechanisms such as sexual selection and sexual conflict have led to the elaboration of traits to gain attention and favours from potential partners. A paradox exists about how coordinated systems can evolve and diverge when there would seem to be a stabilising selection acting. Moth display traits - pheromones - constitute an advantageous model with which to address questions about the evolution of mating systems in animals. Both males and females can possess pheromones that are involved either in close- or longrange communication. Female and male pheromones appear to have different origins and to be under different evolutionary constraints, thus they might be envisioned as independently evolving traits. We conducted laboratory experiments to explore the role of scents released during courtship by males of the European corn borer, Ostrinia nubilalis.
\end{abstract}

Results: Information provided by the male pheromone appears critical for female acceptance. The composition of this male pheromone varies in an age-dependent manner and females show mating preference towards older males in choice experiments. Furthermore, male signals may allow species discrimination and reinforce reproductive isolation. Finally, we found evidence for a genetic correlation between male and female signals, the evolution of which is best explained by the constraints and opportunities resulting from the sharing of gene products.

Conclusion: In this study we used an integrative approach to characterise the male sex pheromone in a moth. Interestingly, the male chemical signal is analogous to the female signal in that structurally similar compounds are being used by both sexes. Hence, in systems where both sexes possess display traits, the pleiotropy of genes generating the traits could influence the evolutionary trajectories of sexual signals and lead to their divergence, with speciation being the ultimate result.

\section{Background}

Understanding the exact ways in which mate choice is exerted is one of the keys to understanding the selective forces driving the processes of assortative mating, reproductive isolation, population divergence and ultimately speciation [1]. Although chemical signalling is one of the major communication channels in animals, few studies have aimed at depicting the importance of scents such as pheromones in mate choice [2-7]. Pheromone bouquets can provide information not only on species and sex, but 
also on age $[5,8]$. Even though evidence for the role of pheromones in mate choice is increasing, the mechanisms are still to a large degree unknown [9]. One of the possible explanations for the evolutionary success of Lepidoptera, the second most species-rich insect order, may lie in the efficiency of their mate-signalling systems. Species-specific sex pheromones emitted by female moths mediate long-range mate attraction of males and play an important role in species recognition $[2,5,10,11]$. In some species, males also possess pheromones that are released at close-range during courtship [5,11-13]. In contrast to the symplesiomorphic nature of the female signalling traits, the male signalling traits in moths are exceedingly variable and labile, suggesting that they have evolved independently on several occasions [11]. The evolution of the male signals has been suggested to constitute an adaptive response in species in which the probability of interspecific mating mistakes is high [14]. Close-range chemical cues have also been proposed as a trait used by females to assess male quality $[3,11,15]$. Among all male characteristics seen as possible cues for female mate preference in insects, age is one that has received much attention. Authors have proposed explanations as to why females might make mate choice decisions [16-20], but few studies have addressed which sensory cues mediate female choice.

The moth sexual communication system is often seen as an example of a coordinated communication system under stabilising selection because females producing atypical pheromone blends and males responding to 'off blends' are usually at a disadvantage compared with typical individuals, producing and responding to the 'population norm' $[10,21]$. Following the asymmetric tracking hypothesis [11], males exert weak selective pressure on conspecific females, and by extension, apply low selective constraints on genes involved in female pheromone biosynthesis. It is noteworthy that male moth pheromones generally consist of volatiles acquired or derived from host plant compounds $[3,11,12,15]$, and thus the pheromone biosynthesis taking place in males normally involves a different enzymatic machinery compared with the de novo biosynthesis leading to female sex pheromones [22]. The contention is that male and female pheromone communication systems have evolved independently.

For various aspects of its ecology and behaviour the European corn borer (ECB), Ostrinia nubilalis (Lepidoptera: Crambidae), offers a unique opportunity for studying female choice in Lepidoptera. The majority of females are monandrous $[23,24]$ and male quality has been demonstrated to be of great importance for female reproductive success [25]. As a consequence of their greater parental investment, females can be expected to be selective in their choice of partner and the overall quality of their mates. Indeed, behavioural observations imply that female choice occurs prior to copulation in ECB $[26,27]$. Male corn borers possess so-called hairpencils located ventrally on the eighth sternite and on the claspers (Fig 1). These organs, normally retracted inside the abdomen, are extruded during the courtship sequence when males are in close vicinity of calling females [26]. In the course of male display, females flick intensively their antennae, suggesting the involvement of male olfactory cues in mate choice, and evidence for male scent to be involved in this mating system has previously been reported [26]. The male pheromone might provide females with a discriminating trait allowing species recognition and/or to assess the overall quality of the males. In addition to the reproductive isolation generated by the male response to female pheromone, the female response to male pheromone would act as a second pre-zygotic barrier reinforcing the reproductive isolation between species. Ethological isolation, caused by female sex pheromone production and perception, is high but not perfect between pheromone strains of the ECB [28-30] and between the ECB and its close relative $O$. furnacalis, the Asian corn borer (ACB). Rare males can be attracted by the pheromone blend of related species or strains, and the formation of hybrids is possible [31-33]. However, the interaction between the so-called $\mathrm{Z}$ and $\mathrm{E}$ strains of $O$. nubilalis in sympatry results in a lower number of hybrids than one would expect and reveals a high level of assortative mating [34,35]. Crossing experi-

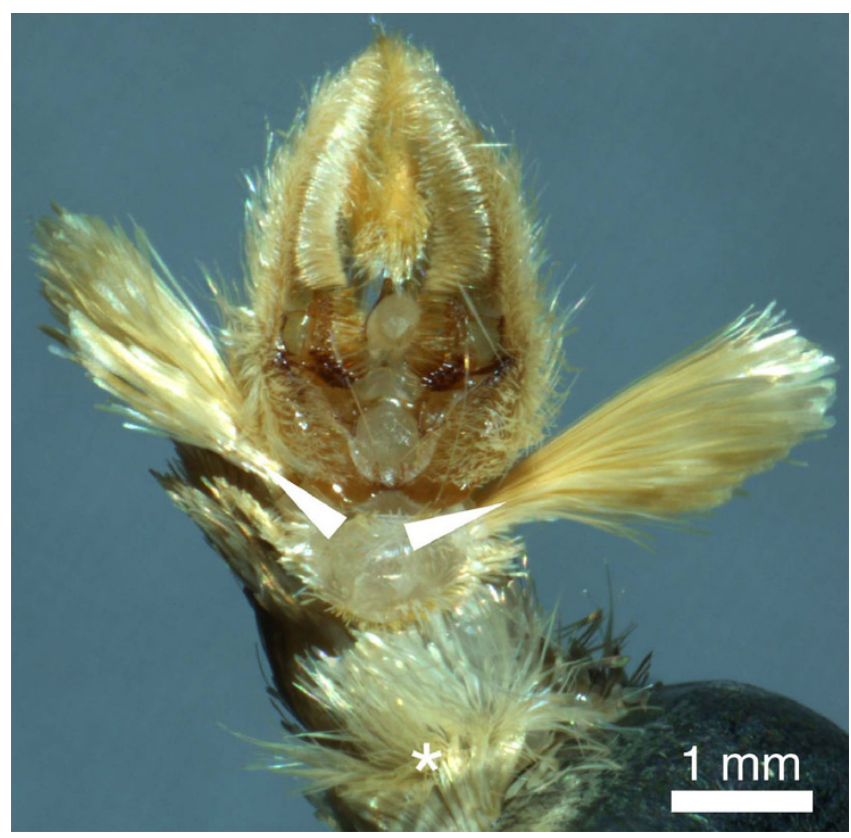

Figure I

Hairpencils of corn borer males. Photograph of the terminal abdominal segments of a male Ostrinia nubilalis with hairpencils exposed. One set is located on the $8^{\text {th }}$ sternite (asterisk) while another set is found on the claspers (arrowheads). (Picture JM Lassance). 
ments revealed the existence of a putative close-range factor determining female preference [36]. Moreover, genetic mapping of the loci causing sexual isolation between pheromone strains of the ECB substantiates the idea that a mechanism different from female sex pheromone prevents gene flow [37]. Comparing the signals produced by males belonging to different species or strains would help to test the hypothesis that male signals promote reproductive isolation.

In many cases, especially when habitat or temporal isolation does not occur, the formation of new species is accompanied by the formation of new pheromones $[5,38]$. Elucidating how new signals arise is a clue to understanding the generation of diversity. In addition, over the years the ECB and its congeners have become an exceptionally interesting model for the study of evolution of moth pheromones and in particular, the evolution of desaturases, which are key biosynthetic enzymes [38]. Compared with its congeners using $\Delta 11$-desaturation in their pheromone biosynthesis to produce $(E)$ - and $(Z)-11$ tetradecenyl acetate and (Z)-9-tetradecenyl acetate, ACB females use a unique mixture of $(E)$ - and $(Z)$-12-tetradecenyl acetate produced through $\Delta 14$-desaturation [39]. The discovery of $\Delta 14$-desaturase transcripts in ECB females gave rise to many questions regarding the origin of this gene $[40,41]$.

In this article, we report on the nature of chemicals associated with the hairpencils displayed by males prior to mating and their role in female choice. ECB females are able to distinguish between males of different ages and the composition of male scents varies accordingly. Including both ECB pheromone strains and the close relative $\mathrm{ACB}$, we compared the chemical profiles of males and found them to differ significantly. The reconstruction of the putative biosynthetic pathway leading to the production of the male pheromone components highlights analogies between males and females. We found the $\Delta 11$ desaturase as well as the unusual $\Delta 14$-desaturase to be expressed not only in females but also in ECB males and to be involved in male pheromone biosynthesis. The emergence of the structurally different female sex pheromone used by ACB may thus have been facilitated by activation of genes involved in male pheromone biosynthesis. Our results suggest that the evolution of pheromone biosynthesis genes in corn borers is best explained by a combination of opportunities and constraints that result from their different functions in males and females.

\section{Results \\ Mating experiments}

We examined whether ECB female mating preference is associated with male age by exposing them to males from three age classes (0-, 2- and 4-day-old males). In a first series of experiments where one female was presented with one male, the proportions of males accepted as mates were significantly different between age classes, females tending to mate more often with older males $\left(\chi^{2}\right.$ $=6.30$, degrees of freedom (df) $=2, P=0.043$ for overall comparison (Figure 2A)). No obvious differences were noticed in male courtship display between age classes. In a second set of experiments, where females were given a choice of three males, the overall analysis indicated a significant difference among age classes, with a differential mating success in favour of older males $\left(\chi^{2}=27.83, \mathrm{df}=\right.$ $2, P<0.001$ for overall comparison (Figure 2B)). Males of all classes approached and courted females. Interestingly, females did not necessarily accept the first courting male. Note that in both experiments there was no influence of age on female mating success $\left(\chi^{2}{ }_{\exp 1}=5.42 ; \chi^{2} \exp 2^{2}=0.786\right.$; $\mathrm{df}=2, P>0.05$ for overall comparison).

\section{Identification of male ECB Z scent}

Since we found 4-day-old males to have a higher mating success, we extracted compounds from the hairpencils of individuals of this age. The composition of male ECB $\mathrm{Z}$ hairpencils was characterised as a blend of 16 carbon chain-length saturated and monounsaturated acetates. Hexadecanyl acetate (16:OAc) and (Z)-9-, $(Z)-11$ - and (Z)-14-hexadecenyl acetates (Z9-16:OAc, Z11-16:OAc and Z14-16:OAc, respectively) were identified according to their mass spectra and retention times. All the compounds showed the $m / z 61$ fragment characteristic of acetates, the ion $[\mathrm{M}-60]$, that is, 224 and 222, respectively for the saturated and unsaturated compounds, as well as all other characteristic fragments of the respective compounds. Double bond positions were confirmed by mass spectral analysis of the dimethyl disulphide (DMDS) adducts, which displayed the characteristic ions of hexadecenyl acetates with double bond in the $\Delta 9(\mathrm{~m} / z 231$, $145,171), \Delta 11(\mathrm{~m} / z 259,117,199)$ and $\Delta 14(\mathrm{~m} / \mathrm{z} 301,75$, 241) position, respectively.

\section{Behavioural assay}

A series of experiments was undertaken to verify the behavioural role of the identified compounds. From a previous study [26], we knew that hairpencil ablation has a critical negative impact on male mating success. We hypothesised that male odour was required for females eventually to accept courting males and that female acceptance could be artificially re-established. First, we confirmed that the ablation of hairpencils has a dramatic effect on males' mating success. Female rejection behaviour was observed as wing flapping to maintain the abdomen inaccessible and then by flying away. Operated males obtained significantly fewer mates than sham-operated ones (Figure 3). Second, we were able to re-establish female acceptance by experimentally adding hairpencil odour or a synthetic mimic. This corroborates the importance of the blend of identified hair- 


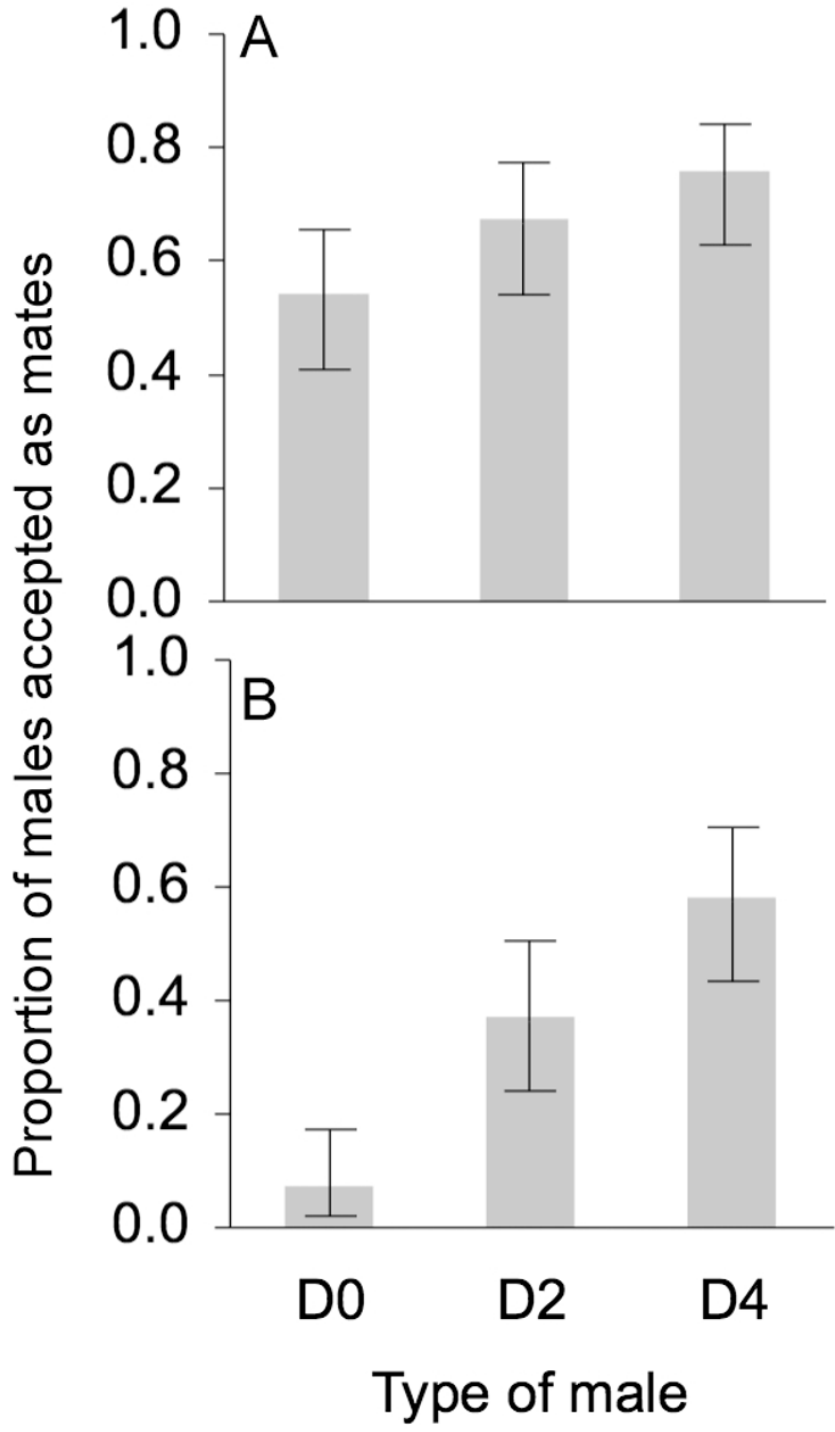

Figure 2

Male mating success in relation to age. Proportion of males accepted as mates for each age class when exposed to females in one-male choice experiment $(A)$ or together with males of other classes (B). Overall comparisons reveal significant differences in the proportion of males accepted as mates among age classes $(P=0.043$ and $P<0.00 I)$. In both series of experiments females tended to mate more frequently with older males. Error bars depict the $95 \%$ confidence limits for the proportions.

pencil odorants to female acceptance and male mating success, and qualifies this male-produced volatile blend as a pheromone. However, the activities of the individual compounds of this blend in influencing female acceptance remains to be verified.

\section{Composition of male ECB Z scent in relation to age}

Differences in male pheromone among age classes could provide a basis for the observed female choice. In some

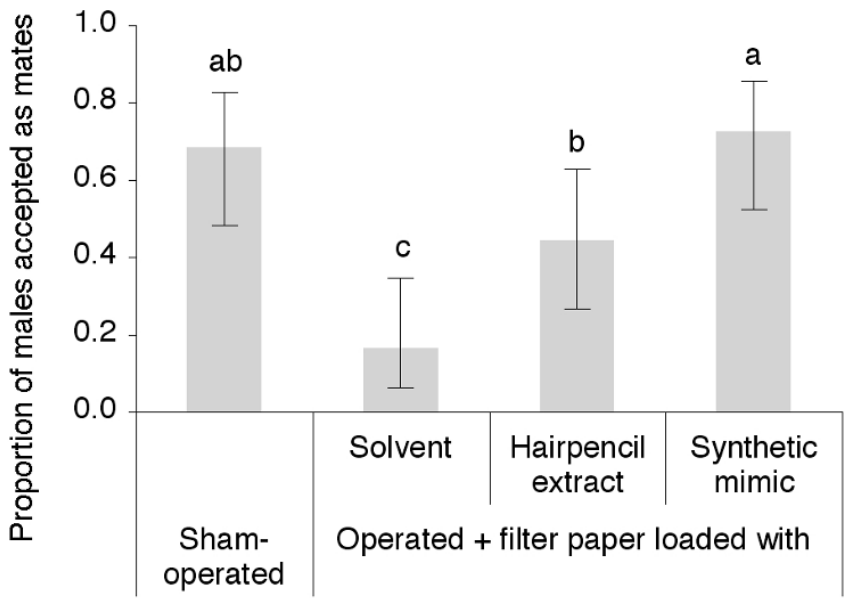

Figure 3

Behavioural demonstration of the role of hairpencil scents. Proportion of males accepted as mates by a female exposed to males sham-operated or with ablated hairpencils plus a filter paper loaded with either solvent (hexane), one male equivalent of 4-day-old male hairpencil extract or a synthetic mimic ( $N=25$ males tested for each category). For each category, no letters in common indicate significant differences at the 0.05 level.

moth species, female preference is correlated with male pheromone content $[3,11,15]$. Therefore, we regressed the pheromone titre (defined as the sum of the four pheromone compounds) on male age to determine whether females could discriminate males on that basis. Pheromone titre did not correlate with male age $\left(r^{2}=0.030, N=\right.$ $45, P=0.258 ; y=2.690+0.078 x ;$ data were natural $\log$ transformed), indicating that this trait does not explain the difference in mating success between age classes. However, females could be sensitive to the composition of the bouquet, and thus we tested whether there was a significant correlation between male age and the proportions of the individual components by use of Spearman's rank correlation coefficients $\left(r_{s}\right)$ (Figure 4 ). The proportions of Z11-16:OAc and 16:OAc were significantly correlated with age, positively in the case of Z11-16:OAc, and negatively in the case of 16:OAc (Figure 4C and 4D).

\section{Comparison of male scent in ECB Z, ECB E and ACB}

We compared hairpencil extracts of ECB Z (French population, the main focus of the present study) with extracts of ECB $Z$ from Hungary and also of the other pheromone strain (ECB E strain from USA and Slovenia) and ACB (China). Gas chromatographic analysis revealed major differences in the composition of the male bouquets (Figure $5 \mathrm{~A}$ ). A discriminant function analysis allowed us to resolve individuals of the three corn borer taxa into three groups (Figure 5B). Male ACB produce a mixture of $\mathrm{Z9}$ 16:OAc and 16:OAc only, and are discriminated from ECB 

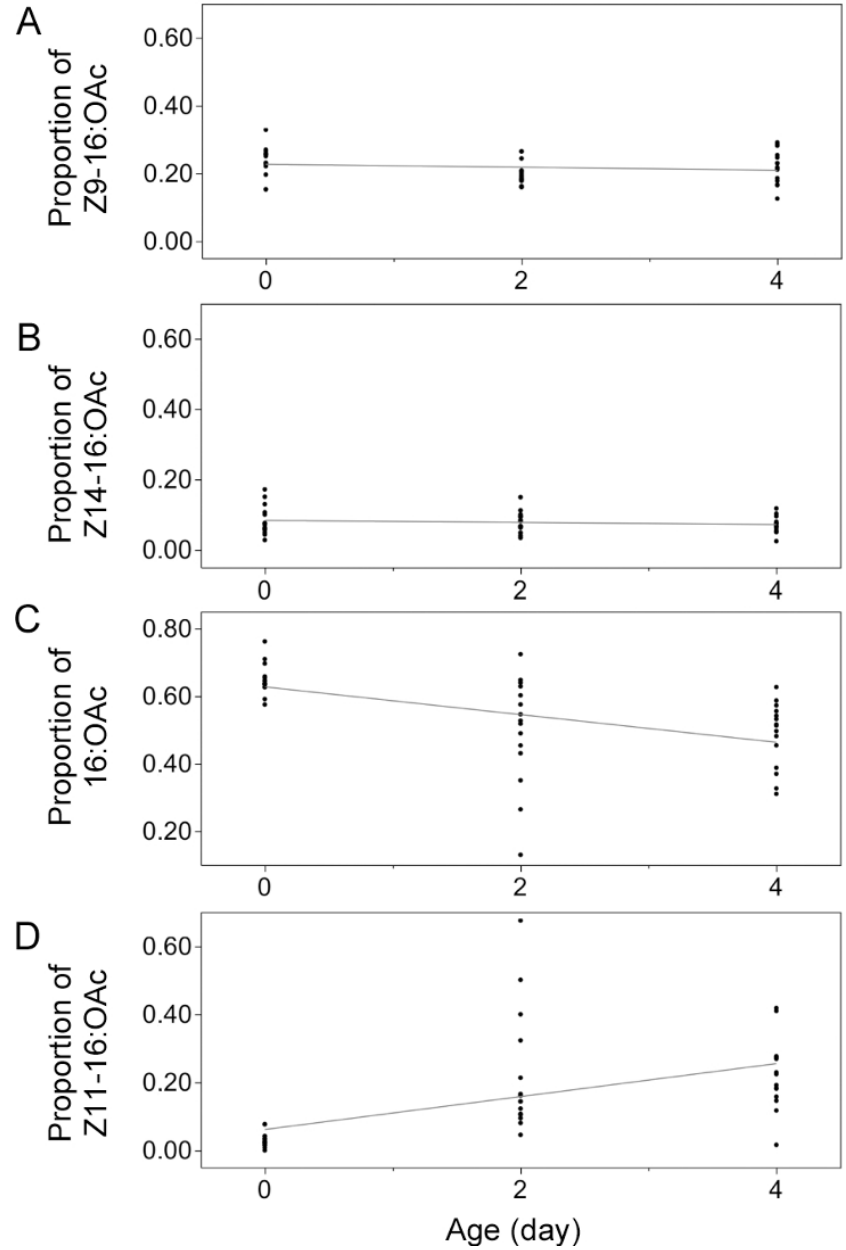

Figure 4

Relationships between the proportion of individual pheromone components and the age of European corn borer $\mathbf{Z}$ males. The proportion of Z9-16:OAc is not correlated with age $(A)\left(r_{s}=-0.157, P=0.302\right)$, neither is the proportion of ZI4-16:OAc (B) $\left(r_{s}=-0.054, P=0.722\right)$. The proportion of $16: \mathrm{OAc}$ is negatively correlated with age (C) $\left(r_{s}=-0.645, P<0.00 \mathrm{I}\right)$, and the proportion of $\mathrm{ZII}-16: \mathrm{OAc}$ is positively correlated with age (D) $\left(r_{s}=0.72 \mathrm{I}, P<0.00 \mathrm{I}\right) . \mathrm{N}=$ 45 males, I 5 per age class.

by the absence of Z14-16:OAc. ECB E individuals differ typically from ECB $Z$ by the low abundance or complete absence of Z11-16:OAc (mean \pm standard error of the mean, $\bar{X}_{\text {ECB E }}=0.36 \pm 0.10 \mathrm{ng}(N=68), \bar{X}_{\text {ECB Z }}=3.09 \pm$ $0.44 \mathrm{ng}(N=72))$. ECB E males producing Z11-16:OAc at levels comparable to ECB $Z$ individuals were rarely found (Figure 5B). As reported above, the blend of young ECB Z (D0) contains a low amount of Z11-16:OAC ( $\bar{X}_{\text {ECB Z D0 }}=$ $0.45 \pm 0.05 \mathrm{ng}(N=24))$, generating an overlap with ЕСВ $\mathrm{E}$ (Figure $5 \mathrm{~B}$ ) and suggesting an incomplete differentiation of the strains for this trait.

\section{Reconstruction of the male pheromone biosynthetic pathway}

As male compounds are structurally similar to the ones constituting the female sex pheromone, we hypothesised that the male pheromone components could be synthesised along a biosynthetic pathway similar to those confirmed in female corn borers ([40] and references therein). Our investigations revealed that, in addition to the ubiquitous fatty acids present (including palmitic and palmitoleic acid, likely precursors of 16:OAc and Z9-16:OAc, respectively), the male extracts contained unsaturated fatty acids identified as (Z)-11-hexadecenoate supposedly resulting from the activity of a $\Delta 11$-desaturase on palmitic acid as well as $(Z)$-and $(E)$-14-hexadecenoate proposed to be produced from the activity of a $\Delta 14$-desaturase. Thus, based on precursor analyses and current knowledge on how pheromones consisting of fatty acid derivatives are produced, we postulated the biosynthetic pathways in Figure 6 leading to male pheromone compounds. This suggests that both sexes could rely on the same $\Delta 11$-desaturase to produce their pheromone components, as this desaturase can introduce double bonds in carbon chains of different length [40]. Also, a $\Delta 14$-desaturase of central importance in the pheromone biosynthetic pathway of female ACB may be part of the male biochemical machinery.

\section{Detection of $\Delta I$ 4- and $\Delta I$ I-desaturase transcripts using reverse transcriptase polymerase chain reaction}

Using gene-specific primers, we analysed by reverse transcriptase polymerase chain reaction (RT-PCR) $\Delta 14$ - and $\Delta 11$-desaturase transcript levels in abdominal tips from 0 , 2- and 4-day-old ECB Z males to confirm the involvement of these enzymes in the male pheromone biosynthesis. The expression of both types of desaturase genes could be detected in male tissues, regardless of age (Figure 7). We also analysed pheromone glands as well as abdomens from newly emerged females. As previously reported [40], the results showed expression of the $\Delta 14$ - and $\Delta 11$-desaturase within the pheromone gland but not in the abdomen (Figure 7). However, it was surprising to find that levels of the two desaturase-encoding transcripts were similarly high. Fatty acid methyl ester extracts from ECB Z female pheromone glands were prepared by base methanolysis and analysed by gas chromatography-mass spectrometry (GC-MS). In addition to the fatty acids common to cell membranes and those resulting from the $\Delta 11$ desaturase activity, the extracts contained unsaturated fatty acids produced by the $\Delta 14$-desaturase $(\mathrm{m} / z 74,87$, 236 [M-32]). Double bond positions were confirmed with DMDS derivatisation, which produced adducts showing characteristic major ions of $\Delta 14$-hexadecenoate $(\mathrm{m} / z 287$, $255,75)$ as well as $\Delta 12$-tetradecenoate $(\mathrm{m} / z 259,227,75)$. Taken together, these results show that $\Delta 14-$ and $\Delta 11$ desaturase genes are transcribed, and their mRNAs effectively translated, in both ECB Z males and females. 

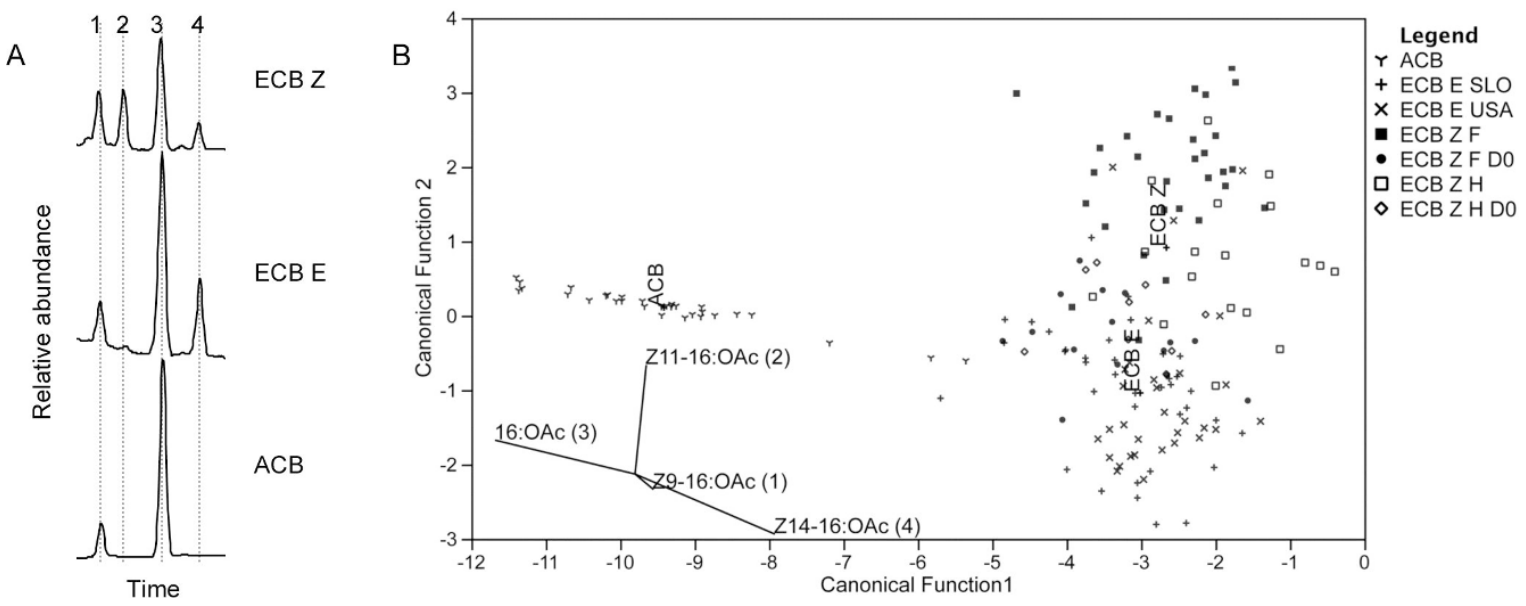

Figure 5

Composition of male pheromone in corn borers. (A) Partial gas chromatograms of hairpencil extracts from virgin 4-dayold ECB Z, ECB E and Asian corn borer (I, Z9-16:OAc; 2, ZII-16:OAc; 3, 16:OAc; 4, ZI4-16:OAc). (B) Plot of canonical discriminant function scores for each species or strain for the first two functions accounting for $89.2 \%$ and $10.8 \%$ of the total variance explained. The four variables were absolute amount of individual compounds, all transformed $\left(Y=\log _{10}(a m o u n t+0.1)\right)$.

\section{Cloning of $\Delta I$ 4- and $\Delta I$ I-desaturases}

To validate the assumption that male and female pheromones are synthesised via shared biochemical pathways, we characterised the $\Delta 14$ - and $\Delta 11$-desaturases expressed in males. We used specific primers designed after the published $\Delta 14$ and $\Delta 11$-desaturase sequences characterised from female pheromone glands [40] to clone the corresponding cDNAs in males. The $\Delta 14$-desaturase transcript possessed nearly identical nucleotide sequence (uncorrected $p$ distance $(p)=0.0034$ and 0.0054 for overall and open reading frame (ORF) comparisons, respectively) and displayed a deduced amino acid sequence identical to the one found in the female pheromone gland. The $\Delta 11$-desaturase transcript was more different at the nucleotide level $(P=0.0329$ and 0.0192 for overall and ORF comparisons, respectively), which corresponds to a deduced amino acid sequence similar at $98.2 \%$ to the sequence previously reported for the female pheromone gland. Since these differences could be the result of difference in insect origin (USA previously versus France in the present study) rather than attributable to PCR errors, we cloned $\Delta 14$ - and $\Delta 11$ desaturase cDNAs from pheromone glands of females obtained from our laboratory culture. When comparing the nucleotide and deduced amino acid sequences of male and female clones, we found that the male clones are identical to the corresponding female clones. The sequences reported in this paper have been deposited in the GenBank database [accession nos. EU350083-EU350086].

\section{Discussion Male pheromone as a display trait determining mating success}

In lepidopterans and birds males are the homogametic sex. This particular feature has been suggested to render these groups of organisms especially conducive to sexual selection and, therefore, to have favoured the elaboration of male secondary sexual characters [42]. Long tails and bright colours generally come to mind but pheromone signals of male moths are certainly equally elaborate display traits [43]. Behavioural observations throughout the reported experiments provide evidence for the ability of ECB females to distinguish between males, and that male pheromone is a secondary sexual trait associated with female mating preference. In our laboratory setting, male mating success is related to age. Although we cannot dismiss the potential existence of intrinsic differences in aggressiveness and persistence between the age classes, which has been demonstrated in butterflies [44], the results of our experiments demonstrate that there is a correlation between the age of a male and the composition of his pheromone. We propose that female mating preference in ECB is associated with the odour bouquet released by males. A role of male pheromone also in male-male interactions cannot be excluded but was beyond the scope of this study.

Our demonstration of pheromone-based female mate choice and identification of a male-produced courtship pheromone in ECB is of particular importance because it may alter our understanding of the role of pheromones in corn borer speciation. The comparison of male pheromones of the three corn borer taxa indicates a potential species-specific function of these male pheromones. Differences in female sex pheromone between the $\mathrm{E}$ and $\mathrm{Z}$ strains of ECB have been insufficient to establish a perfect reproductive barrier between these siblings. Pheromones of ECB $Z$ and $E$ malesdiffer noticeably in the presence or absence of Z11-16:OAc. Therefore, male pheromone 


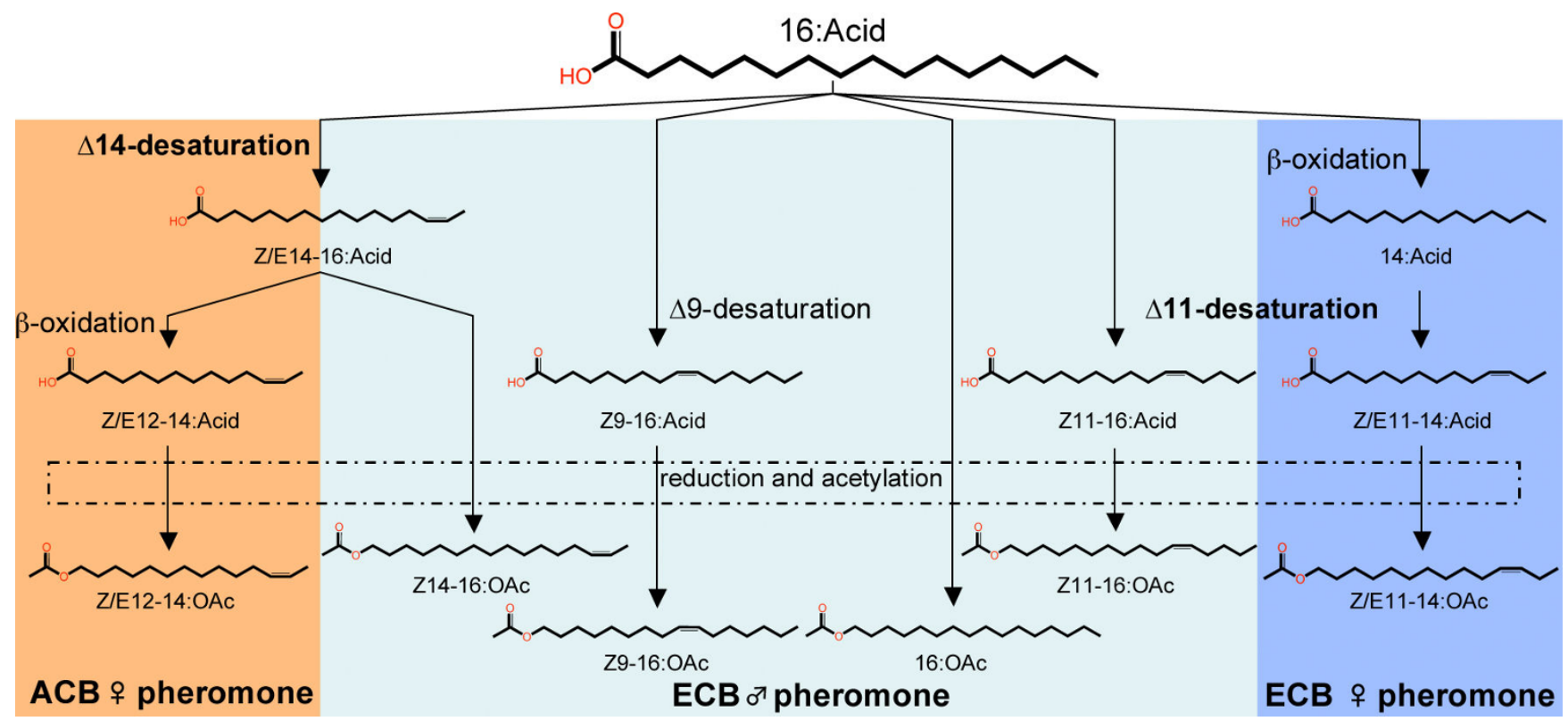

\section{Figure 6}

Proposed pheromone biosynthetic pathways for the European corn borer and Asian corn borer. The male and female pheromone components in the European corn borer (ECB) and the Asian corn borer (ACB) are biosynthesised de novo from the ubiquitous palmitic acid (I6:Acid). The routes leading to the I4-carbon acetate components produced by females and to the I6-carbon acetate components used by males employ common key desaturation enzymes. The $\Delta$ I4-desaturase appears to be common to female ACB and male ECB pathways, as well as the $\triangle \mathrm{II}$-desaturase that is shared in male and female ECB pathways. Note that the $\Delta$ 9-desaturation leading to palmitoleic acid (Z9-I6:Acid) also occurs in females of both species, but the reduction and acetylation of this substrate occur in males only. Pathways for male compounds postulated from the analysis of precursors and for female compounds adapted from [40] and references therein.

could act to reinforce reproductive isolation. The evolution of mate choice in ECB $\mathrm{Z}$ females may have been partly driven by this difference, since choosing (old) males whose blend contains Z11-16:OAc would result in less hybrid mating. Obviously, future investigations using more populations will be required to elucidate fully this issue. In addition, our results suggest that the male signal could act as an indicator of genetic and/or resource benefits and provide females with information on the quality of the courting males within populations. Thus, female mating preference oriented towards the oldest male in our experiments would be adaptive, females preferring these males because they have proven survival capabilities and may possess a fit genotype for their environment.

\section{The consequences of sharing gene products on evolution of the display trait}

The male corn borer pheromone components are structurally similar to those produced by females and their biosynthesis appears to involve enzymes common to both sexes (Figures 6 and 7). This finding has important consequences for our current view of the evolution of moth pheromones and communication systems in general. First, it may be that genes expressed to generate signalling traits in both sexes have increased functional constraints at a greater number of sites. For example, functional con- straints might have arisen on the $\Delta 11$-desaturase that was demonstrated [40] to produce a mixture of $(Z)-11$ - and (E)-11-tetradecenoic acids, which is used in biosynthesis of the female sex pheromone, as well as (Z)-11-hexadecenoic acid, which is used in males (Figure 6). Evidence from previous studies suggests that breadth of gene expression and degree of specialisation influence evolutionary rate $[45,46]$. Indeed, the $\Delta 11$-desaturase gene was found to have a slower evolutionary rate compared with the more specialised $\Delta 14$-desaturase gene [38]. Also, the sharing of the $\Delta 11$-desaturase gene to produce $(Z)-11$ hexadecenoic acid, which is relevant in a male context, and (Z)-11- and (E)-11-tetradecenoic acids, precursors of the female sex pheromone, may cause antagonistic selection on the enzyme to generate only the type of substrate used in the context of a certain sex. Intralocus sexual conflict will potentially result since the two sexes are under disruptive selection upon a shared character but are genetically constrained from evolving in different directions [47]. Hence, pheromone signals, such as several other display traits, are often found with associated structures that may have evolved through sexual conflict of interest [43]. The sexually antagonistic coevolution driven by this intralocus conflict could be resolved by the evolution of sex limitation, that is, the expression of the enzyme by one sex only, or by the evolution of insensitivity of the female 


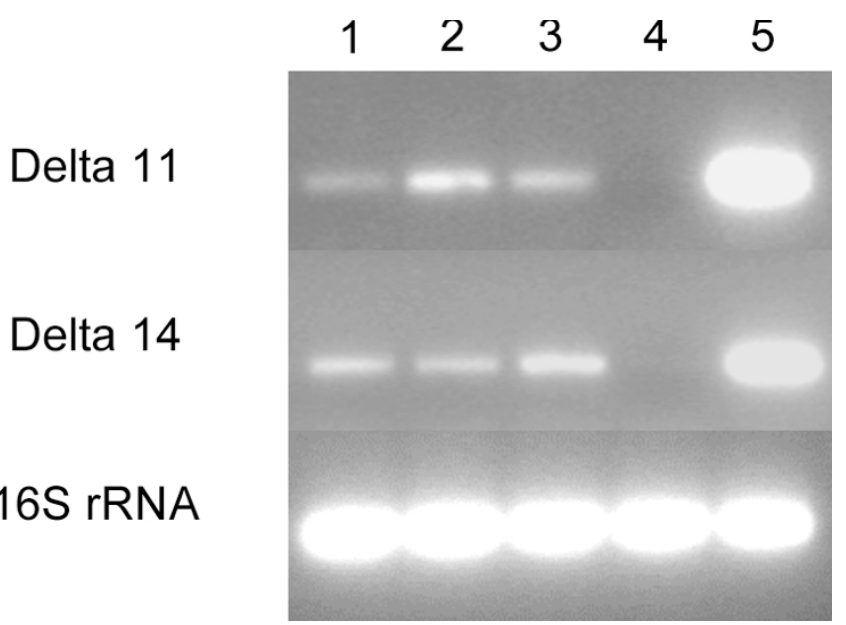

Figure 7

Reverse transcriptase polymerase chain reaction of $\Delta I I-$ and $\Delta I$ 4-desaturase transcripts from adult European corn borer tissues. I, 2 and 3: abdominal tip from 0, 2- and 4-day-old males, respectively; 4: abdomen (terminal segments excluded) from newly emerged females; 5 : pheromone gland from newly emerged females. Expression of I6S rRNA indicated as control.

to the male stimulus [48]. This would, however, lead the females into a 'sensory trap' from which it is difficult for them to escape, because if the $\Delta 11$-desaturase is not involved in the biosynthesis of the male pheromone or if females evolve insensitivity, they also lose the naturally selected benefit of their preference as Z11-16:OAc allows them to distinguish between age classes and/or ensures the distinction between $\mathrm{Z}$ and $\mathrm{E}$ males.

The existence of a genetic correlation between male and female signals can also be the source of evolutionary opportunities. Genes implicated in one sex's trait could serve in the context of the other sex's trait, leading to the emergence of a new signal. The presence of individuals responding to this new signal could help its maintenance and generates suitable conditions for divergence and possibly speciation to occur. Our results suggest an answer to the intriguing question as to why a sex pheromone biosynthesis gene that does not function to produce, for instance, the ECB female sex pheromone, would be maintained in the genome over long-term evolutionary time $[40,41]$. Our findings imply that the $\Delta 14$-desaturase gene in question is functional in males and involved in the biosynthesis of a putative male pheromone component (Z14-16:OAc) (Figure 6). It can be speculated that the processing of the $\Delta 14$ gene as a new functional desaturase gene occurred in males of an ancestral Ostrinia species. Members of the same gene subfamily were found in the Drosophila melanogaster genome, suggesting that these genes have derived from a duplication event that took place before the split between Diptera and Lepidoptera
[40]. However, the presence of $\Delta 14$-desaturase or its products have only been reported in the lepidopteran genus Ostrinia. It is tempting to place such rarity in parallel with the polyphyletic nature of male-pheromone systems [11]. Interestingly, in addition to the expected $\Delta 11$ transcripts, a high level of $\Delta 14$-desaturase transcripts was noticed in the female pheromone gland. Firm evidence for the enzyme to be active in that tissue was brought by the detection of fatty acids unsaturated at the fourteenth position. Trace amounts of $\Delta 12$-tetradecenoic acids were also detected in the pheromone gland of the ECB $\mathrm{Z}$ female. Produced through one step of limited $\beta$-oxidation, these acids constitute the precursors for the female pheromone of the ACB [49] (Figure 6). This suggests that the differences between ECB $Z$ male and female pheromones may not be the result of differential activity of the desaturase systems but rather of differences at other steps in the biosynthesis. A major shift in the expression pattern is likely to have occurred in an ancestral Ostrinia population and given rise to the ACB $[40,41]$. Under such a scenario, some females would have started to emit a pheromone blend consisting of a mixture of $\Delta 11$ - and $\Delta 12$-tetradecenyl acetates [50]. The emergence of the new blend, and subsequent tracking by responsive males, would have been facilitated by the pre-existence of enzymes linked to a biosynthetic pathway involving $\Delta 14$-desaturation and olfactory receptors devoted to the detection of Z14-16:OAc. Our results thus identify a mechanism that facilitates the rapid evolution of pheromones by large saltational shifts. Parallel to the pleiotropy of genes producing the signal may be the pleiotropy of genes responsible for signal detection and interpretation. Investigations are under way to determine the existence of a link between male and female pheromone reception.

\section{Conclusion}

One of the conundrums for evolutionary biologists is how coordinated communication systems under stabilising selection can still diverge and lead to the process of speciation $[1,5]$. The revealed diversity of male pheromone systems [12] is likely to be parallel to diversity in genes generating the associated traits. Indeed, what we describe above could have occurred in other genera or families and, therefore, have significantly influenced the evolution of female pheromone production in many lepidopterans. Additionally, our findings show how new sexual traits could emerge from changes in expression pattern and set the scene for speciation. Further studies should aim at elucidating the mechanisms that generate such changes and how new traits can become fixed.

\section{Methods \\ Moths}

Insects were obtained from laboratory cultures reared on a maize-wheat germ diet. Eggs or pupae from the different strains were obtained from: ECB $\mathrm{Z}$, France (French 
National Institute for Agricultural Research (INRA), UE Entomologie, Poitou-Charentes, France) and Hungary (T Dekker, Swedish University of Agricultural Sciences (SLU), Alnarp, Sweden); ECB E, USA (WL Roelofs, Cornell University, Ithaca, USA) and Slovenia (T Dekker, SLU); ACB, China (CH Zhao, Academia Sinica). Males and females were separated before eclosion and placed in different climate chambers maintained at $23 \pm 1{ }^{\circ} \mathrm{C}$ in a 17 hour:7-hour light-dark photoperiod. Newly emerged adults were separated daily and considered to be 0-dayold.

\section{Mating experiments}

We compared the mating success of males in relation to age. To test this we defined three age classes $(0-, 2-, 4$-dayold males) and set up two experiments in which females were in the presence of either one or three males of each class. ECB Z males and females were placed together into a cylindrical container (1 litre) with a source of water 1 hour before the onset of scotophase. After the onset of scotophase and during the entire 7-hour scotophase, events in the mating enclosure were monitored at intervals of 15 minutes in order to check courtship behaviour and mating occurrence. In the first series of experiments, we subjected each female to a one-male choice test $(N=$ 60 individuals for each of the three male classes, giving a total of 180 females), scoring the formation of mating pairs. For each age class we calculated the proportion of males accepted as mates (number of pairs observed/ number of males tested). A $\chi^{2}$ test was used to test the dependence of male mating success on age. In the second set of experiments, 60 females were permitted a choice between three males, one from each age class. Each male was anaesthetised and marked on the thorax using a paint marker pen and given a colour corresponding to his age class. Colours were rotated between trials and had no detectable effect on male mating success. For each mating pair observed $(N=47)$, we noted the age class of the male. The proportion of males accepted as mates for each age class was calculated as the number of males of a given class accepted as mates over the number of mating pairs observed. Again, a $\chi^{2}$ test was used to test the dependence of male mating success on age. Equal numbers of 0-, 2- or 4-day old females were used in the experimental design of both experiments. The $95 \%$ confidence intervals of individual proportion were computed according to the method described by Newcombe [51] using the online tool available at Vassarstats [52].

\section{Identification of male ECB Z scent}

Volatiles were extracted by placing hairpencils in hexane and recovering the solvent after 1 hour at room temperature. The samples were analysed on a GC (Hewlett-Packard 5890) connected to a MS (Hewlett-Packard 5972, EI $70 \mathrm{eV}$ ). A HP-1MS column (methyl siloxane, $30 \times 0.25$ $\mathrm{mm}, \mathrm{df}=0.25 \mu \mathrm{m}$, Agilent Technologies) was used and the oven temperature was maintained at $50^{\circ} \mathrm{C}$ for 2 minutes and then programmed at $10^{\circ} \mathrm{C}$ per minute to $250^{\circ} \mathrm{C}$ kept for 10 minutes (carrier gas helium, velocity $30 \mathrm{~cm} /$ second). The compounds were identified by comparison of their spectra with standard mass spectra and retention times. Confirmation of double bond position was obtained by DMDS derivatisation and subsequent GC-MS analysis [53].

\section{Behavioural assay}

In a one-male choice assay, we exposed females to 4-dayold males from which hairpencils had been ablated surgically on the day preceding the experiments. In order to facilitate hairpencil ablation, males were anaesthetised with carbon dioxide. Hairpencils were extruded by applying gentle pressure on the abdomen and trimmed with fine forceps to remove as much as possible. The same procedure was used on the sham-operated males with no removal of the hairpencils. The assays were conducted in a chamber consisting of a glass cylinder $(13 \mathrm{~cm}$ diameter $\times 25 \mathrm{~cm}$ height) with steel screening covering the open ends. Airflow was generated by placing the arena in a wind tunnel together with the addition of a small fan. First, one calling female moth (0- to 4-day-old) with the pheromone gland exposed was introduced into the arena and placed upwind from the males. Single 4-day-old virgin males were then added downwind. Each male was given 10 minutes to mate successfully with the female. Odour replacements were made by introducing an odour source (filter paper) upwind from the female while the male was courting. The odour source was loaded with either 4-dayold male hairpencil extract (one male equivalent) or a synthetic blend mimicking the odour of those males. The synthetic mimic consisted of a blend of 20\% Z9-16:OAc, 15\% Z11-16:OAc, 53.5\% 16:OAc and 11.5\% Z14-16:OAC corresponding to $4 / 3 / 11 / 2$ ng of individual compound in $20 \mu \mathrm{l}$ of hexane. Positive and negative controls consisted of sham-operated males and operated males plus a filter paper with the solvent alone applied on to it. The trial was ended either when successful coupling was observed (the male was considered 'accepted as mate') or at the end of the allotted time. Each treatment was tested using 25 males. All the males included in the statistical analysis displayed and attempted to copulate with females. The proportions of males accepted as a mate in individual treatments were compared using $z$-tests $(\alpha=0.05)$.

\section{Composition of male scent in relation to age and taxa}

To characterise hairpencil pheromone titre and composition in relation to age (ECB Z) and taxa (ECB Z and E and $\mathrm{ACB})$, hairpencil volatiles were extracted in heptane containing a known amount of pentadecanyl acetate chosen as internal standard. Samples were analysed on a GC (Hewlett-Packard 5890) equipped with a flame ionisation 
detector. A HP-1MS column was used and the oven temperature was maintained at $80^{\circ} \mathrm{C}$ for 2 minutes and then programmed at $10^{\circ} \mathrm{C}$ per minute to $250^{\circ} \mathrm{C}$ kept for 10 minutes (carrier gas helium, velocity $60 \mathrm{~cm} /$ second).

\section{Statistical analyses}

All statistical tests were carried out in SPSS 16 software with the exception of the multivariate analysis of variation in pheromone components (absolute amounts in ng) of ECB $\mathrm{Z}$ and $\mathrm{E}$ and ACB males performed by canonical discriminant function analysis using JMP 7 software.

\section{Precursor analysis}

Fatty acid methyl ester extracts were prepared by base methanolysis. Total lipid extraction was performed by immersing ECB Z male abdominal tips in $100 \mu \mathrm{l}$ of chloroform:methanol $(2: 1 \mathrm{v} / \mathrm{v})$. After 1 hour, the tissues were removed and a gentle stream of nitrogen was applied to evaporate the solvent. Conversion of fatty acyl moieties to methyl esters was made by treating the samples with 100 $\mu \mathrm{l}$ of potassium hydroxide $(0.5 \mathrm{M}$ in methanol). The reaction was ended after 1 hour by the addition of $100 \mu \mathrm{l}$ of hydrochloric acid (1.0 M in water). The fatty acyl methyl esters were recovered in hexane and the samples subsequently analysed by GC-MS. Double bond positions were confirmed by DMDS derivatisation.

\section{Collection of insect tissue and RNA extraction}

Male abdominal tips were carefully dissected from $0-, 2-$ and 4-day-old virgin male moths and stored at $-80^{\circ} \mathrm{C}$. Pheromone glands and abdomens from 0-day-old virgin female moths were dissected and stored similarly. Total RNA was isolated and purified from dissected tissues using the TRIzol reagent (Invitrogen) according to the manufacturer's recommended procedures.

\section{Cloning and sequence analysis of $\Delta I 4-$ and $\Delta I I$ - desaturases from male and female}

Based on the publicly available sequence information ( 111 : AF441221; $\Delta 14$ : AF441220), specific primers were designed to obtain sequence information corresponding to the $\Delta 14$ - and $\Delta 11$-desaturases expressed in male and female $O$. nubilalis $\mathrm{Z}$ (primer sequences reported in Table $1)$.

Total RNA (100 ng) was used to amplify a fragment corresponding to the ORF of the $\Delta 14$-desaturase gene. Two primers ( $\Delta 14$-ORF-s plus $\Delta 14$-ORF-as) were used with the Superscript III One-Step RT-PCR kit (Invitrogen) following the manufacturer's instructions. Amplification products were analysed by electrophoresis on agarose gel. An amplification product of around 1250 base pairs was excised from the gel, purified using the Qiagen gel extraction kit (Qiagen) and cloned using TOPO TA cloning kit with PCR2.1-TOPO vector and One Shot TOP10 chemically competent Escherichia coli (Invitrogen) for sequencing. Subsequently, primers were designed to obtain 5'and 3 '-ends by rapid amplification of cDNA ends $(\Delta 14$ 5'RACE and $\triangle 14-3$ 'RACE, respectively). We used the SMART RACE cDNA amplification kit (Clontech) following the manufacturer's protocol. The coding sequences were deduced with the sequencing result of the 5 '-end, central region and 3 '-end.

For the $\Delta 11$-desaturase, two primers were designed to generate overlapping amplification products by rapid amplification of cDNA ends ( $\triangle 11-5$ 'RACE for the 5 '-region and $\Delta 11-3$ 'RACE for the 3'-region). The coding sequences were deduced with the sequencing result of the 5 '- and 3 '-ends.

\section{RT-PCR}

Total RNAs (50 ng) were used to amplify fragments of the $\Delta 14$ - and $\Delta 11$-desaturase genes using the Superscript III One-Step RT-PCR kit (Invitrogen) following the manufacturer's instructions. The gene-specific primer sets used were $\Delta 11$-RT-s and $\Delta 11$-5'RACE for $\Delta 11, \Delta 14$-ORF-s and $\Delta 14-5$ 'RACE for $\Delta 14$. PCR products were analysed on a $1 \%$ agarose gel and visualised with ethidium bromide.

\section{Abbreviations}

16:OAc: hexadecanyl acetate; ACB: Asian corn borer; df: degrees of freedom; DMDS: dimethyl disulphide; ECB:

Table I: Primers used for polymerase chain reaction and reverse transcriptase polymerase chain reaction amplification.

\begin{tabular}{|c|c|c|}
\hline Gene & Primer & Sequence (5' to $\left.3^{\prime}\right)$ \\
\hline \multirow[t]{4}{*}{$\Delta / 4$} & $\Delta \mathrm{I} 4-\mathrm{ORF}-\mathrm{s}$ & CCCAGCAAACATGGCAGACATAGAC \\
\hline & $\Delta \mathrm{I} 4-\mathrm{ORF}-\mathrm{as}$ & CGCGAAACTTCTACGCACCGTAACTT \\
\hline & $\triangle I 4-5$ 'RACE & CGCTCATGTCAATGGTCTTCCTCAGCTT \\
\hline & $\triangle I 4-3 ' R A C E$ & ATGAACAAAGGAGACGGCACTTACGAGG \\
\hline \multirow[t]{3}{*}{$\Delta I I$} & $\triangle \mathrm{II}-5$ 'RACE & GCCAAGCACAGCAAGATTCAGAAGAACG \\
\hline & $\triangle \mathrm{II}-3^{\prime} \mathrm{RACE}$ & GATTGGAATCACTGCTGGTGCCCATAGA \\
\hline & $\Delta \mathrm{II}-\mathrm{RT}-\mathrm{s}$ & GGCATTTCAAAATACTGCGCTCTCGTGG \\
\hline \multirow[t]{2}{*}{ I6S rRNA } & $16 \mathrm{~S}-\mathrm{s}$ & TGAAGGGCTGCAGTATTTTG \\
\hline & I6S-as & TCGAGGTCGCAAACTCTTTT \\
\hline
\end{tabular}


European corn borer; GC: gas chromatography; MS: mass spectroscopy; ORF: open reading frame; RT-PCR: reverse transcriptase polymerase chain reaction; Z9-16:OAc: Z1116:OAc, Z14-16:OAc, (Z)-9-, (Z)-11- and (Z)-14-hexadecenyl acetate, respectively.

\section{Authors' contributions}

JML participated in the design of the study, carried out the experiments, performed statistical analyses and drafted the manuscript. CL financed, mentored and participated in the design of the study and helped to draft the manuscript. Both authors read and approved the final manuscript.

\section{Acknowledgements}

We thank EV Jirle for insect rearing assistance, M Liénard for her helpful advice, GP Svensson, D Hasselquist, AP Rooney, RT Cardé and BO Bengtsson for helpful comments. Special thanks to R Lindelöf from LRI Instruments $A B$ in Lund for his great help with hairpencil pictures. This work was supported by grants from the Swedish Research Council for Environment, Agricultural Sciences and Spatial Planning (FORMAS) and the Swedish Research Council (VR).

\section{References}

I. Coyne JA, Orr HA: Speciation Sunderland: Sinauer; 2004

2. Andersson M: Sexual Selection Princeton: Princeton University Press; 1994.

3. Eisner T, Meinwald J: The chemistry of sexual selection. Proc Natl Acad Sci USA 1995, 92:50-55.

4. Jones TM, Hamilton JGC: A role for pheromones in mate choice in a lekking sandfly. Anim Behav 1998, 56:89|-898.

5. Wyatt TD: Pheromones and Animal Behaviour: Communication by Smell and Taste Cambridge: Cambridge University Press; 2003.

6. Martin J, Lopez P: Links between male quality, male chemical signals, and female mate choice in Iberian Rock lizards. Funct Ecol 2006, 20:1087-1096.

7. Nieberding CM, de Vos H, Schneider MV, Lassance JM, Estramil N Andersson J, Bång J, Hedenström E, Löfstedt C, Brakefield PM: The male sex pheromone of the butterfly Bicyclus anynana: Towards an evolutionary analysis. PloS ONE 2008, 3:e275I.

8. Vane-Wright $\mathrm{VI}$, Boppré $\mathrm{M}$ : Visual and chemical signalling in butterflies: functional and phylogenetic perspectives. Philos Trans R Soc Lond B Biol Sci 1993, 340:197-205.

9. Johansson BG, Jones TM: The role of chemical communication in mate choice. Biol Rev 2007, 82:265-289.

10. Löfstedt C: Moth pheromone genetics and evolution. Philos Trans R Soc Lond B Biol Sci 1993, 340:167-177.

II. Phelan LP: Evolution of mate-signaling in moths: phylogenetic considerations and predictions from the asymmetric tracking hypothesis. In The Evolution of Mating Systems in Insects and Arachnids Edited by: Choe JC, Crespi B]. Cambridge: Cambridge University Press; 1997:240-256

12. Birch MC, Poppy GM, Baker TC: Scents and eversible scent structures of male moths. Annu Rev Entomol 1990, 35:25-58.

13. Hillier NK, Vickers NJ: The role of heliothine hairpencil compounds in female Heliothis virescens (Lepidoptera: Noctuidae) behaviour and mate acceptance. Chem Senses 2004, 29:499-5II.

14. Phelan LP, Baker TC: Evolution of male pheromone in moths: reproductive isolation through sexual selection? Science 1987 235:205-207.

15. Löfstedt C, Vickers NJ, Roelofs WL, Baker TC: Diet related courtship success in the Oriental fruit moth, Grapholita molesta (Tortricidae). Oikos 1989, 55:402-408.

16. Zuk M: Parasite load, body size, and age of wild-caught male field crickets (Orthoptera: Gryllidae): Effects on sexual selection. Evolution 1988, 42:969-976.
17. Hansen TF, Price DK: Good genes and old age: Do old mates provide superior genes? J Evol Biol 1995, 8:759-778.

18. Jones TM, Balmford A, Quinnel RJ: Adaptative female choice for middle-aged mates in a lekking sandfly. Proc $R$ Soc Lond B Biol Sci 2000, 267:68I-686

19. Beck CW, Powell LA: Evolution of female mate choice based on male age: Are older males better mates? Evol Ecol Res 2000 , 2:107-I 18.

20. Wedell N, Ritchie MG: Male age, mating status and nuptial gift quality in a bushcricket. Anim Behav 2004, 67: 1059-1065.

21. Butlin R: Genetic variation in mating signals and responses. In Speciation and the Recognition Concept: Theory and Application Edited by: Lambert DM, Spencer HG. Baltimore: Johns Hopkins University Press; 1995:327-366.

22. Eisner T, Meinwald J: Alkaloid-derived pheromones and sexual selection in Lepidoptera. In Insect Pheromone Biochemistry and Molecular Biology Edited by: Blomquist G], Vogt RG. London: Elsevier Academic Press; 2003:341-368.

23. Fadamiro HY, Baker TC: Reproductive performance and longevity of female European corn borer, Ostrinia nubilalis: effects of multiple mating, delay in mating, and adult feeding. J Insect Physiol 1999, 45:385-392.

24. Torres-Vila LM, Jennions MD: Male mating history and female fecundity in the Lepidoptera: do male virgins make better partners? Behav Ecol Sociobiol 2005, 57:318-326.

25. Royer L, McNeil JN: Male investment in the European corn borer, Ostrinia nubilalis (Lepidoptera: Pyralidae): Impact on female longevity and reproductive performance. Funct Ecol 1993, 7:209-2I5.

26. Royer L, McNeil JN: Evidence for a male sex pheromone in the European corn borer, Ostrinia nubilalis (Hübner) (Lepidoptera: Pyralidae). Can Entomol 1992, I 24: I I3-II6.

27. Schlaepfer MA, McNeil JN: Are virgin male lepidopterans more successful in mate acquisition than previously mated individuals? A study of the European corn borer, Ostrinia nubilalis (Lepidoptera: Pyralidae). Can J Zool 2000, 78:2045-2050.

28. Klun JA, Chapman OL, Mattes KC, Wojtkowski PW, Beroza M, Sonnet $\mathrm{PE}$ : Insect sex pheromones: minor amount of opposite geometrical isomer critical to attraction. Science 1973 , |81:661-663.

29. Kochansky J, Cardé RT, Liebherr J, Roelofs WL: Sex pheromone of the European corn borer, Ostrinia nubilalis (Lepidoptera: Pyralidae), in New York. J Chem Ecol 1975, I:225-231.

30. Glover TJ, Tang XH, Roelofs WL: Sex pheromone blend discrimination by male moths from $E$ and $Z$ strains of European corn borer. J Chem Ecol 1987, I 8: |43-I5I.

31. Roelofs WL, Glover T, Tang XH, Sreng I, Robbins P, Eckenrode C, Löfstedt C, Hansson BS, Bengtsson BO: Sex pheromone production and perception in European corn borer moths is determined by both autosomal and sex-linked genes. Proc Natl Acad Sci U S A 1987, 84(2I):7785-7789.

32. Linn CE, O'Connor M, Roelofs WL: Silent genes and rare males: a fresh look at pheromone blend response specificity in the European corn borer moth, Ostrinia nubilalis. J Insect Sci 2003. 3:15.

33. Linn CE, Musto Cl, Roelofs WL: More rare males in Ostrinia: response of Asian corn borer moths to the sex pheromone of the European corn borer. J Chem Ecol 2007, 33:199-2I 2.

34. Cardé RT, Roelofs WL, Harrison RG, Vawter AT, Brussard PF, Mutuura A, Munroe E: European corn borer: pheromone polymorphism or sibling species? Science 1978, 199:555-556.

35. Klun JA, Huettel MD: Genetic regulation of sex pheromone production and response: interaction of sympatric pheromonal types of European corn borer, Ostrinia nubilalis (Lepidoptera: Pyralidae). J Chem Ecol 1988, I4:2047-206I.

36. Pélozuelo L, Meusnier S, Audiot P, Bourguet D, Ponsard S: Assortative mating between European corn borer pheromone races: beyond assortative meeting. PLOS ONE 2007, 2:e555.

37. Dopman EB, Bogdanowicz SM, Harrison RG: Genetic mapping of sexual isolation between $E$ and $Z$ pheromone strains of the European corn borer (Ostrinia nubilalis). Genetics 2004, 1 67:301-309.

38. Baker TC: Mechanism for saltational shifts in pheromone communication systems. Proc Natl Acad Sci USA 2002, 99:13368-13370. 
39. Ishikawa Y, Takanashi T, Kim CG, Hoshizaki S, Tatsuki S, Huang Y: Ostrinia spp. in Japan: their host plants and sex pheromones. Entomol Exp Appl 1999, 91 1:237-244.

40. Roelofs WL, Liu W, Hao G, Jiao H, Rooney AP, Linn CE: Evolution of moth sex pheromones via ancestral genes. Proc Natl Acad Sci USA 2002, 99:13621-13626.

41. Roelofs WL, Rooney AP: Molecular genetics and evolution of pheromone biosynthesis in Lepidoptera. Proc Natl Acad Sci USA 2003, 100:9179-9/84.

42. Reeves HK, Pfenning DW: Genetic biases for showy males: Are some genetic systems especially conducive to sexual selection? Proc Natl Acad Sci USA 2003, 100:1089-1094.

43. Hill GE: Trait elaboration via adaptive mate choice: sexual conflict in the evolution of signals of male quality. Ethol Ecol Evol 1994, 6:35I-370.

44. Fisher K, Perlick J, Galetz T: Residual reproductive value and male mating success: older males do better. Proc $R$ Soc Lond $B$ Biol Sci 2008, 275: I5 17-I524.

45. Drummond DA, Bloom JD, Adami C, Wilke CO, Arnold FH: Why highly expressed proteins evolve slowly. Proc Natl Acad Sci USA 2005, I 02: | 4338-| 4343.

46. Koonin EV, Wolf YI: Evolutionary systems biology: links between gene evolution and function. Curr Opin Biotechnol 2006, 17:48|-487.

47. Pischedda A, Chippindale AK: Intralocus sexual conflict diminishes the benefits of sexual selection. PloS Biol 2006, 4:e356.

48. Chapman T: Evolutionary conflicts of interest between males and females. Curr Biol 2006, I6(I 7):R744-754.

49. Zhao $\mathrm{CH}$, Löfstedt $\mathrm{C}$, Wang $\mathrm{X}$ : Sex pheromone biosynthesis of the Asian corn borer, Ostrinia furnacalis (II). Biosynthesis of $(E)$ - and (Z)- I 2-tetradecenyl acetate involves $\Delta$ - I4 desaturation. Arch Insect Biochem Physiol 1990, I 5:57-65.

50. Cardé RT, Haynes KF: Structure of the pheromone communication channel in moths. In Advances in Insect Chemical Ecology Edited by: Cardé RT, Millar JG. Cambridge: Cambridge University Press; 2004:283-332.

51. Newcombe RG: Two-sided confidence intervals for the single proportion: comparison of seven methods. Stat Med 1998, 17:857-872.

52. Vassarstats: Web Site for Statistical Computation [http://fac ulty.vassar.edu/lowry/VassarStats.html]

53. Buser HR, Arn H, Guerin P, Rauscher S: Determination of double bond position in mono-unsaturated acetates by mass spectrometry of dimethyl disulfide adducts. Anal Chem 1983, 55:818-822.

Publish with Bio Med Central and every scientist can read your work free of charge

"BioMed Central will be the most significant development for disseminating the results of biomedical research in our lifetime. "

Sir Paul Nurse, Cancer Research UK

Your research papers will be:

- available free of charge to the entire biomedical community

- peer reviewed and published immediately upon acceptance

- cited in PubMed and archived on PubMed Central

- yours - you keep the copyright
BioMedcentral 